\title{
Electromagnetic calorimeter prototype for the SoLID project at Jefferson Lab
}

\author{
Y. Tian ${ }^{1}$, J.-P. Chen ${ }^{2}$, C. Feng ${ }^{1 \star}$, J. Jiao ${ }^{1}$, A. Li $^{1}$, Y. Yu ${ }^{1}$, and X. Zheng ${ }^{3}$ \\ ${ }^{1}$ Shandong University, Jinan 250100, China \\ 2 Thomas Jefferson National Accelerator Facility, Newport News, VA 23606, USA \\ ${ }^{3}$ University of Virginia, Charlottesville, VA 22904, USA
}

\begin{abstract}
SoLID (Solenoidal Large Intensity Device) is a large acceptance spectrometer which can handle very high luminosity, being planned for experimental Hall A at Jefferson Lab, USA. The shashlik-type sampling detector will be used for the electromagnetic calorimeter for SoLID. This calorimeter is 18 radiation-lengths long with 194 layers each of $1.5 \mathrm{~mm}$-thick plastic scintillators alternating with $0.5 \mathrm{~mm}$-thick lead plates. A few prototype of the calorimeter have been built at Shandong University. the light yield of these modules have been tested with cosmic ray. The assembling process of these prototypes and cosmic ray test results are presented.
\end{abstract}

Keywords: SoLID, Electromagnetic Calorimeter, Shashlik Calorimeter

\section{Introduction}

To fully exploit the potential of the Jlab $12 \mathrm{GeV}$ energy upgrade, a large acceptance spectrometer known as $\operatorname{SoLID(Solenoidal~Large~Intensity~Device)[1],~which~can~handle~very~high~luminosity,~is~}$ being planed in Hall A at Jefferson Lab. As shown in Figure 1, SoLID project includes two configurations: the SIDIS (Semi-Inclusive Deep Inelastic Scattering) and the PVDIS (Parity-Violating Deep Inelastic Scattering), which have different geometrical layouts but similar detectors. SoLID Electromagnetic Calorimeter (EC) will be used in both PVDIS and SIDIS configurations to separate electron from pions and other hadrons by measuring their energy deposition. Special challenges due to high background and high magnetic field must be considered in the EC design. The desired performance of SoLID EC is illustrated in Table 1.

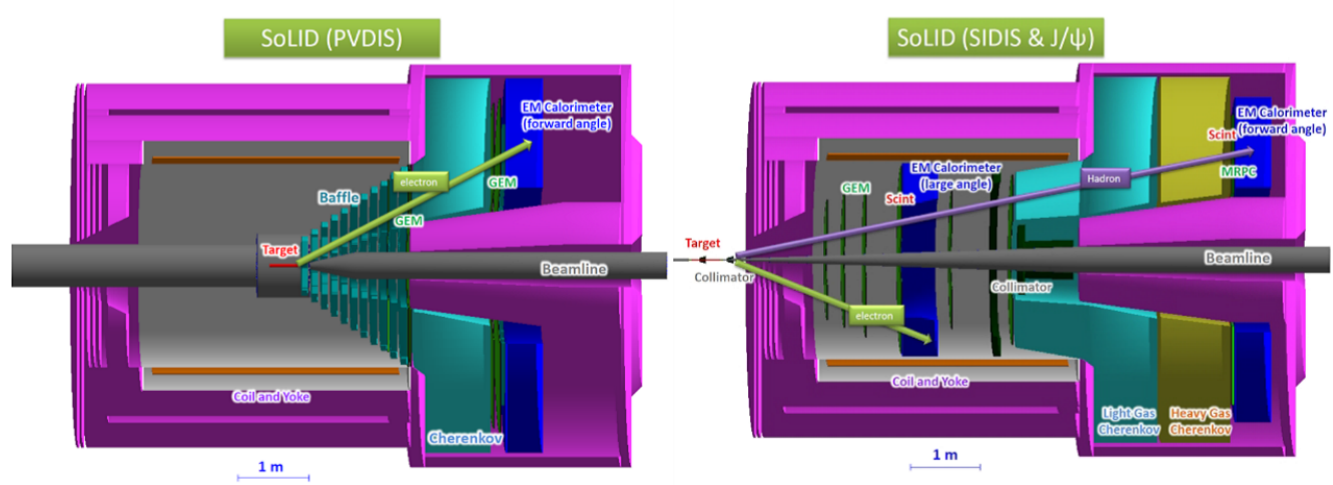

Figure 1: Two configurations of the SoLID detector layout. The detectors in blue are the Electromagnetic Calorimeters.

A shashlik style[2] sampling EC is designed to meet the needs of both configurations. The Shashlik calorimeter is based on a technique to readout the scintillation light of multi-layer lead/scintillator plate sampling calorimeter with the use of WLS (Wave-Length Shifting) fibers running perpendicular

\footnotetext{
${ }^{\star}$ Corresponding author. E-mail: fengcf@sdu.edu.cn
} 


\begin{tabular}{|c|c|}
\hline Specification & Desired performance \\
\hline$\pi^{-}$ & $50-100: 1$ \\
\hline$e^{-}$efficiency & $>90-95 \%$ \\
\hline Energy resolution & less than $10 \% / \sqrt{E}(\mathrm{GeV})$ \\
\hline Timing resolution & $100 \sim 500 \mathrm{ps}$ \\
\hline Radiation resistance & $>500 \mathrm{kRad}$ \\
\hline Position resolution & $<1 \mathrm{~cm}$ \\
\hline
\end{tabular}

Table 1: SoLID EC desired performance.

to the plates through holes in the plates. It has been applied in several particle and nuclear physics experiments, such as COMPASS, LHCb and ALICE. Scintillation light is absorbed, re-emitted and transported through WLS fibers and clean fibers to photon detector as EC readout signal. A new shashlik EC design, shown in Figure 2, was simulated to reach the required goals. To provide a good balance among position resolution, background and the budget, the lateral size of calorimeter module is optimized to $100 \mathrm{~cm}^{2}$ of hexagon shape.

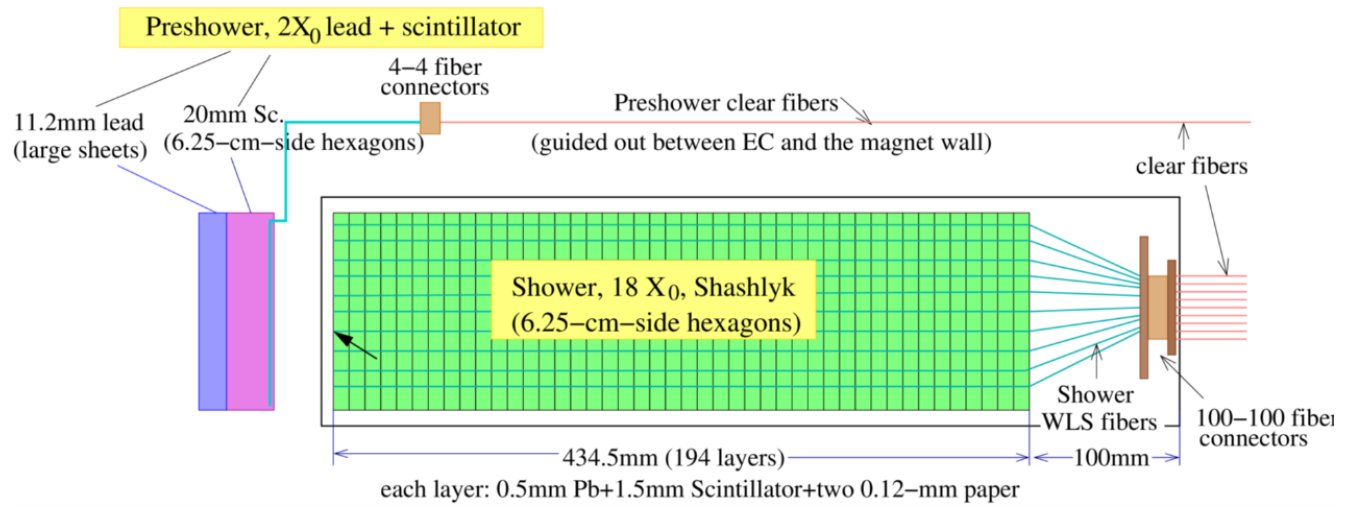

Figure 2: Shashlik EC detector design. It is segmented to preshower and shower detectors longitudinally to enhance particle identification. The shower detector is 18 radiation-lengths long with 194 layers each of $1.5 \mathrm{~mm}$-thickness plastic scintillators alternating with $0.5 \mathrm{~mm}$-thickness lead plates. Both detectors are read out by Photo-Multiplier Tubes(PMTs).

Based on the new shashlik design, the research presented in this article will focus on the assembly and tests of the prototypes. To optimize module quality and light yield, the choice of material and construction as well as preliminary results of cosmic ray tests for both individual material and the whole shashlik module will be presented.

\section{Material Selection of EC Prototype}

Energy resolution is a key performnce parameter of calorimeter. The photon statistics is an importance source contributing to the calorimeter resolution. To achieve a lower statistics uncertainty, Number of Photon-Electrons(NPE) collected by a PMT should be as many as possible. The photo-electron yield[3] depends not only on the lead and the scintillator, but also on light collection efficiency. These issues must be investigated fully to optimize photon statistics.

\subsection{Plastic Scintillator and Reflector Layer Material Test}

Two batches of scintillator plates with different scintillation formulations from the same company were used separately in the assembly of shashlik module. We use special setup which includes five layers of reflector-scintillator-reflector-lead structure to test the performance of scintillator plates. 
The test result indicates the second batch of scintillators with improved formulation has a light yield $26 \%$ better than the first batch and will be used in the prototypes.

Several materials with high reflectivity were considered as the reflective layer between scintillator and lead plates. Although they all have good reflectivity(above 90\%), light decreases rapidly in thin scintillator layers after several reflections if the reflectivity is low. The reflector test[4] is performed as the same as the scintillator test method. The test result is shown in Table 2. The result shows the Tyvek paper has the best light yield, which is twice of the case with no reflector.

\begin{tabular}{|c|c|c|c|c|c|}
\hline Reflector material & No reflector & Printing paper & Aluminum foil & Tyvek paper & MCPET \\
\hline Relative light yield value & $0.85 \pm 0.02$ & $1.06 \pm 0.06$ & $0.97 \pm 0.08$ & $1.61 \pm 0.16$ & $1.24 \pm 0.05$ \\
\hline
\end{tabular}

Table 2: Relative light yield for different reflector material. All relative results are compared with another reference paper reflector.

\subsection{WLS Fiber Selection and the Processing on Fiber}

WLS fiber is the light transportation media between the detector and PMT. Two kinds of $1 \mathrm{~mm}-$ diameter WLS fibers are considered in the prototype: Y11(Kuraray) and BCF91A(Saint-Gobain). Both fibers have double cladding, and similar absorption and emission spectrum. Test result shows the BCF91A has the similar light yield as the Y11 without bending and the BCF91A is chosen for the shower detector because of its lower price.

In order to couple all fibers to PMT in alignment, fibers must be polished and processed in bundles. Optical cement is used to glue the fibers together and then the end of fiber bundle is cut and polished by a high-speed rotation milling cutter with both high efficiency and good quality.

For polishing of the other end of fiber which is insered into holes of module seperately, a special freezing method is applied. This method uses a urethane holder to keep fibers in bundle and freeze the holder and fibers in liguid nitrogen before polishing. Silver mirror layer applying magnetron sputtering technique[5] is used as reflector at this end with both good reflectivity and uniformity . Test result shows that silver plating mirror provides more than $70 \%$ improvement on light collection comparing with no mirror.

\section{Prototype Assembly and Prototype}

As shown in Figure 3, a prototype assembly tool is designed to satisfy the need of aligning nearly 800 pieces of sheets and also compressing the whole module to avoid gap between sheets. It can provide at least $5000 \mathrm{~N}$ pressure which is monitored by three pressure sensors.

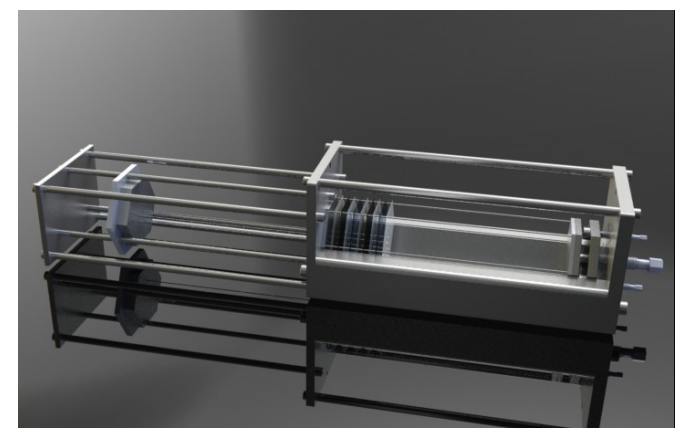

Figure 3: Assembly tool for shashlik shower module.

To improve light collection and also provide light shielding from other modules, the shashlik modules must be coated with painting reflector. The painting used on the module sides is a mixture 
of $\mathrm{TiO}_{2}$ and glue. With a 1:1 mixing ratio, the light yield is $29 \%$ better compared to the module without painting.

Three shashlik prototypes with different materials were assembled successfully at Shandong University. The prototype module built later has improved material and reflection. The materials used in three prototypes are listed in Table 3 . All three prototypes are tested by cosmic ray, and the test results are also shown in Table 3.

\begin{tabular}{|c|c|c|c|c|c|c|}
\hline Prototype No. & WLS fiber & Fiber reflector & Scintillator & Painting & Reflector layer & Test result(NPE) \\
\hline$\# 1$ & BCF91A & No reflector & Original & TiO2+glue & Paper & 212.5 \\
\hline$\# 2$ & BCF91A & Silver mirror & Enhanced & TiO2+glue & Paper & 413.8 \\
\hline$\# 3$ & Y11 & Silver mirror & Enhanced & TiO2+glue & Paper & 484.5 \\
\hline
\end{tabular}

Table 3: Three shashlik prototypes material list. The improvement between \#2 and \#1 is the sintillator and adding fiber mirror reflector. The improvement between \#3 and \#2 is the fiber and painting.

\section{Shashlik Shower Prototype Cosmic Ray Test}

The cosmic ray test was performed to study the light yield property of the shashlik shower prototype. The dominant energy loss of cosmic ray, which are mostly muons, is ionization instead of electromagnetic shower. However the output signal still indicates other general property of calorimeter, especially the light yield.

The test setup sketch is shown in Figure 4 (left). Shashlik detector signal is readout by PMT with specific gain[6] calibrated from SPE(single photon-electron) spectrum. The NPE is calculated according to the ratio between the total charge and the SPE charge by the following equation:

$$
\mathrm{NPE}=\mathrm{Q} /(\mathrm{e} \times \text { Gain })
$$

where $\mathrm{Q}$ is charge acquired from $\mathrm{QDC}($ Charge to Digital Converter) with pedestal subtracted and $\mathrm{e}$ is the charge of a single electron.
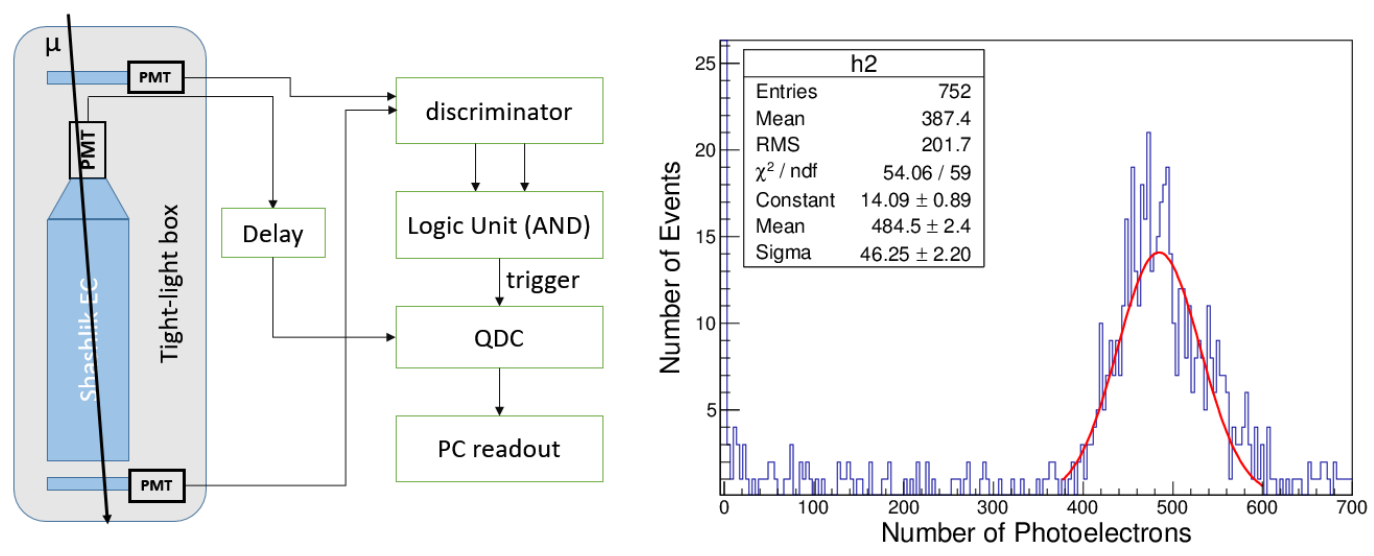

Figure 4: Left: Sketch of shashlik prototype cosmic ray test setup. Two hexagon scintillators that have the same lateral section as shashlik module are used as trigger to make sure muons penetrate all shashlik layers. Right: Prototype \#3 NPE test result.

The cosmic ray test result of \#3 prototype is shown in Figure 4 (right). NPE spectrum is fitted by a Gaussian function. The prototype with better material and reflection shows an improving light yield, and so far the best result is \#3 prototype which shows a light yield of 484 photo-electrons in vertical test. 


\section{Conclusions and Outlook}

The shashlik shower prototype is assembled successfully following the design based on simulation and the experience from other experiment. Cosmic ray test results show good light yield of shashlik

prototype with improved material. For the next step, we will continue working on material selection and ensuring the quality of assembly. Most importantly, to acquire the energy resolution of prototype, beam test using electrons with known energies will be performed in the future.

\section{References}

1. SoLID Collaboration et al. Solid preliminary conceptual design report. Technical report, Jefferson Lab: http://hallaweb. jlab. org/12GeV/SoLID/dowload/doc/solid_precdr. pdf, 2014.

2. GS Atoian, GI Britvich, SK Chernichenko, S Dhawan, VV Issakov, OV Karavichev, TL Karavicheva, VN Marin, AA Poblaguev, IV Shein, et al. An improved shashlyk calorimeter. Nuclear Instruments and Methods in Physics Research Section A: Accelerators, Spectrometers, Detectors and Associated Equipment, 584(2):291-303, 2008.

3. AV Arefev, IM Belyaev, BM Bobchenko, KI Voronchev, AI Golutvin, OB Gushchin, V Yu Egorychev, IE Korolko, TG Kvaratskheliya, IV Machikhilyan, et al. A study of light collection in shashlik calorimeters. Instruments and Experimental Techniques, 51(4):511-522, 2008.

4. Ang LI, Si-yuan FENG, Zhong-quan ZHANG, and Jian-bin JIAO. Optical performance studying for the eflector used in shashlyk electromagnetic calorimeter. Nuclear Electronics $\&$ Detection Technology, 8:873, 2016.

5. P Cortese, T Peitzmann, AP de Haas, GJL Nooren, CJ Oskamp, A van den Brink, CG Ivan, R Kamermans, PG Kuijer, MAJ Botje, et al. Alice electromagnetic calorimeter technical design report, 2008.

6. Xu Wang, Zhong-Quan Zhang, Ye Tian, Yan-Yan Du, Xiao Zhao, Fu-Wang Shen, Chang-Yu Li, YanSheng Sun, and Cun-Feng Feng. Setup of a photomultiplier tube test bench for lhaaso-km2a. Chinese physics C, 40(8):086003, 2016. 